\title{
Des souris, \\ des rats et des \\ hommes
}

> La recherche biomédicale est considérée par nos sociétés comme une nécessité et les réflexions sur les moyens à mettre en œuvre pour la développer s'accordent sur le constat que: «à défaut de pouvoir expérimenter sur l'homme, l'expérimentation animale est indispensable»[1]. Celle-ci, pour être légitime, doit respecter la fameuse règle des 3R (Raffiner, Remplacer, Réduire) énoncée dès 1959 par Russell et Burch [2]. En effet, bien que permettant certaines approches moléculaires, expérimentales ou modélisées, les méthodes alternatives conservent un caractère réducteur et ne permettent pas d'appréhender l'ensemble d'un organisme au sein de son environnement. À ce jour, il reste donc encore indispensable d'utiliser des modèles animaux pour générer des connaissances valides en recherche fondamentale et appliquée. La recherche fait ainsi appel à une grande variété d'organismes-modèles, parmi lesquels les rongeurs (rats et souris) sont les plus utilisés: en France, en 2016, 59,6\% des animaux utilisés pour la recherche étaient des souris et $8,9 \%$ étaient des rats [3]. Le propos de cet article est de montrer en quoi les rongeurs sont des modèles expérimentaux importants et de donner quelques exemples des connaissances nouvelles qu'ils ont apportés. <

L'appartenance des rongeurs à la classe des mammifères les rend proches de l'homme, même s'ils n'en partagent pas toutes les caractéristiques. Parmi les mammifères, leurs propriétés zootechniques (petite taille, reproduction rapide et en grand nombre) leur confèrent une qualité indéniable d'organismes-modèles, et ils sont largement répandus dans les divers laboratoires de par le monde. Le Tableau / en est un témoignage, avec la longue liste des Prix Nobel dont les découvertes ont été obtenues grâce aux modèles rongeurs, le premier étant celui de Nicolle en 1928. II a été suivi en 1939 par celui décerné à Domagk pour la découverte du

Vignette (Photo @ Hélène Gilgenkrantz).

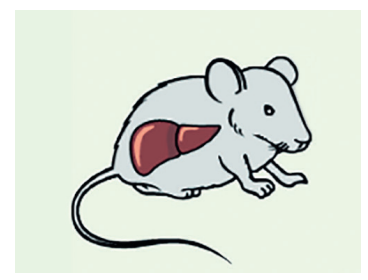

En quoi les modèles

rongeurs restent

indispensables pour

la production de

connaissances

Hélène Hardin-Pouzet ${ }^{1}$, Serban Morosan ${ }^{2}$

'Sorbonne Université, UM 119, Inserm UMRS 1130, CNRS UMR 8246, Neuroscience Paris Seine, Institut de Biologie Paris Seine, 7, quai Saint-Bernard, 75005 Paris, France.

${ }^{2}$ Sorbonne Université, UMS 28, Inserm, Faculté de Médecine, F-75013 Paris, France. helene.pouzet@upmc.fr

premier antibiotique commercialement exploitable, le sulfamidochrysoïdine (ou Prontosil ${ }^{\circledR}$ ), qui a été rapidement supplanté par la pénicilline, ayant valu elle-même le Prix Nobel en 1945 à Fleming, Flore et Chain !

\section{Le modèle souris}

La souris (Mus musculus) est actuellement l'organisme-modèle le plus utilisé en recherche biomédicale. Elle présente de nombreux intérêts : sa taille (10 cm, 35 grammes en moyenne), sa courte durée de vie (de l'ordre d'un an), son rythme rapide de reproduction (3 à 8 petits par portée et une gestation de 21 jours) et son faible coût par rapport à d'autres organismes-modèles. Il est ainsi possible d'élever des colonies de taille suffisante et dans un laps de temps raisonnable pour obtenir des résultats ayant une réelle validité statistique. La génétique de la souris est étudiée depuis longtemps (un des pères de la génétique murine est le français Lucien Cuénot, 1866-1951) et de nombreux mutants spontanés sont disponibles. L'isolement de lignées consanguines ou isogéniques a permis de réduire la variation naturelle entre les sujets et, à l'inverse, de mettre en évidence l'importance du fonds génétique. C'est le cas par exemple de cette étude de toxicologie réalisée par Church et al. démontrant l'importance du fonds génétique dans la toxicité d'un extrait du thé vert [4]. Le séquençage complet du génome de la souris, en 2002, a démontré la proximité phylogénétique des souris et des hommes: $90 \%$ des gènes humains ont un équivalent chez la souris, permettant d'élaborer des approches génétiques et fonctionnelles valides. Lorsque les gènes orthologues produisent des effets différents entre souris et homme, le 


\begin{tabular}{|c|c|c|}
\hline Année & Lauréats & Thèmes \\
\hline 1928 & C. Nicolle & Pathogenèse du typhus \\
\hline 1939 & G. Domagk & Effets antibactériens du Pronostil ${ }^{\circledR}$ \\
\hline 1943 & E.A. Doisy, H. Dam & Découverte de la fonction de la vitamine $K$ \\
\hline 1945 & A. Fleming, દ.B. Chain, H. Florey & Découverte de la pénicilline \\
\hline 1951 & M. Theiler & Vaccin contre la fièvre jaune \\
\hline 1954 & J.F. Enders, T.H. Wellers, F.C. Robbins & Vaccin contre la poliomyélite \\
\hline 1964 & K.દ. Bloch, F. Lynen & Régulation du métabolisme du cholestérol et des acides gras \\
\hline 1966 & F.P. Rous, C.B. Huggins & Virus induisant des tumeurs et traitement hormonaux des cancers \\
\hline 1968 & R. Holley, G. Khorana, M. Nirenberg & Interprétation du code génétique et synthèse des protéines \\
\hline 1970 & J. Axelrod, B. Katz, U. von Euler & Physiologie des neurotransmetteurs \\
\hline 1974 & A. Claude, C. de Duve, G. Palade & Organisation fonctionnelle et structurale des cellules \\
\hline 1975 & H.M. Ternin, R. Dulbecco, D. Baltimore & Interactions entre les virus oncogènes et le génome \\
\hline 1980 & J. Dausset, J.D. Snell, B. Benaceraff & Description et fonction du complexe majeur d'histocompatibilité \\
\hline 1982 & S.K. Bergström, B.I. Samuelsson, J.R. Vane & Découverte des prostaglandines \\
\hline 1984 & N. Jerne, G. Köhler, C. Milstein & Principe de production des anticorps monoclonaux \\
\hline 1985 & M.S. Brown, J.L. Goldstein & Découverte de la régulation du métabolisme du cholestérol \\
\hline 1986 & S. Cohen, R. Levi-Montalcini & $\begin{array}{l}\text { Facteur de croissance des nerfs (NGF) et facteur de croissance épidermique } \\
(\varepsilon G F)\end{array}$ \\
\hline 1987 & S. Tonegawa & Principes génétiques à l'origine de la diversité des anticorps \\
\hline 1988 & J. Black, G.B. Bion, G.H. Hitchings & $\begin{array}{l}\text { Découverte des facteurs les plus importants pour la conception des médica- } \\
\text { ments }\end{array}$ \\
\hline 1996 & P. Doherty, R. Zinkernagel & Reconnaissance par le système immunitaire de cellules infectées par un virus \\
\hline 1997 & S. Prusiner & Découverte des prions \\
\hline 1999 & G. Blobel & $\begin{array}{l}\text { Découverte des signaux intrinsèques des protéines permettant leur localisation } \\
\text { et leur transport dans la cellule }\end{array}$ \\
\hline 2000 & A. Carlsson, P. Greengard, E.R. Kandel & Transduction du signal nerveux \\
\hline 2004 & R. Axel, L.B. Buck & Récepteurs olfactifs et organisation du système olfactif \\
\hline 2007 & M.R. Capecchi, M.J. Evans, 0. Smithies & $\begin{array}{l}\text { Principe de l'introduction spécifique d'une modification génétique, cellules } \\
\text { embryonnaires souches }\end{array}$ \\
\hline 2008 & H. zur Hausen, F. Barré-Sinousi, L. Montagnier & Découverte des papillomavirus et du virus de l'immunodéficience humaine \\
\hline 2009 & દ. Blackburn, C. Greider, J. Szostak & Protection des chromosomes par les télomères et la télomérase \\
\hline 2010 & R. Edwards & Développement de la fécondation in vitro \\
\hline 2011 & J. Hoffmann, B. Beutler, R. Steinman & Système immunitaire inné et cellules dendritiques \\
\hline 2012 & J.B. Gurdon, S. Yamanaka & Reprogrammation des cellules pour devenir pluripotentes \\
\hline 2014 & J. O’Keefe, M.B. Moser, E.I. Moser & Centre GSP du cerveau et positionnement dans l'espace \\
\hline
\end{tabular}

Tableau I. Prix Nobel de physiologie ou médecine ayant utilisé des modèles murins. 
remplacement dans le génome du gène de la souris par son équivalent humain permet cependant la création de lignées humanisées. Ainsi, les souris SCID (severe combined immunodeficiency) et leurs dérivées, toutes immunodéficientes, ont permis le développement des modèles humanisés, notamment pour la production d'anticorps monoclonaux humains à visée thérapeutique, permettant, entre autres, une bonne prise de greffe lors de transplantation chez l'homme ${ }^{1}$. L'infectiologie et la parasitologie sont aussi des domaines qui font appel à des modèles de souris humanisées grâce à la construction de lignées susceptibles à l'infection par certains micro-organismes, comme la bactérie Listeria monocytogenes, les virus de l'hépatite $B$ et C (HBV, HCV) ou encore le parasite Plasmodium falciparum [5].

Le développement des outils de transgenèse et la possibilité de cultiver des cellules-souches totipotentes (cellules souches embryonnaires ou CSE) ont permis de créer de nombreux mutants de type knock-in (intégration) ou knock-out (délétion) : 10000 gènes avaient ainsi été inactivés en 2010 et plus 21000 en 2012. Le prix Nobel de 2007 a d'ailleurs récompensé Capecchi, Evans et Smithies pour leurs travaux sur la recombinaison homologue et les CSE. Le raffinement, ces dernières années, des technologies de recombinaison, de type Cre-lox ${ }^{2}$ ou CRISPR/Cas9 (clustered regularly interspaced short palindromic repeat) [24] $(\rightarrow)$ et de transgenèse inductible, renforce encore l'intérêt de ces modèles. On peut citer comme exemple récent la mise au point de

$(\rightarrow)$ Voir la Nouvelle de H. Gilgenkrantz, $\mathrm{m} / \mathrm{s}$ $n^{\circ}$ 12, décembre 2014, page 1066

modèles bioluminescents, permettant de tester des agents anti-microbiens contre des infections résistantes aux antibiotiques classiques [6]. Le domaine de la génétique n'est pas le seul dans lequel le modèle souris se révèle performant: les différents systèmes de son organisme présentent une organisation et une régulation proches de celles de l'homme. Également, les souris sont susceptibles de contracter certaines maladies humaines telles que le diabète, certains cancers, des troubles liés à l'anxiété, etc. Ainsi, de nombreux modèles des maladies d'Alzheimer, de Creutzfeldt-Jakob, de Parkinson ont été développés. Ils ont par exemple permis de démontrer l'implication de l'alpha-synucléine dans l'étiologie de la maladie de Parkinson. Ils permettent également de tester de nouvelles préparations galéniques, comme ce modèle d'étude des effets anesthésiques de préparation de liposomes sur un bloc nerveux périphérique [7]. Dans le domaine des réponses inflammatoires et immunitaires, un modèle de souris permettant la déplétion transitoire des neutrophiles a conduit à de nouvelles avancées dans la compréhension des mécanismes du choc endotoxinique [8] $(\rightarrow)$.

La cancérologie est un champ d'utilisation de $\rightarrow$ Voir la Synthèse de C.M. Gillis et L.L. Reber, $\mathrm{m} / \mathrm{s} \mathrm{n}^{\circ} 4$, avril 2018, page 339 modèles souris nécessitant des caractéristiques particulières : ils doivent permettre le développement des tumeurs avec une haute pénétrance et

\footnotetext{
${ }^{1}$ Les anticorps monoclonaux faits chez des souris humanisées ne proviennent pas de SCID humanisées mais de souris dont une grande partie des gènes codant les Ig de souris ont été invalidés et dont le génome contient une grande partie des gènes orthologues humains. Les Ac Mc utilisés pour la prévention du rejet de greffe sont les anti-CD25 et l'anti-CD3 développés bien avant l'apparition de souris humanisées.

${ }^{2}$ Une version classique du système Cre-lox consiste à croiser une souris exprimant Cre dans une population spécifique de cellules (lignée Cre) avec une souris dite « rapportrice », dans laquelle l'expression d'un transgène codant une protéine d'intérêt est sous le contrôle d'un promoteur à forte activité transcriptionnelle. En amont de la séquence codante se trouve un codon STOP flanqué de sites loxP («floxé ») qui empêche la transcription du transgène. Dans les souris issues de ce croisement (la génération Fl), le codon STOP est excisé uniquement dans les cellules exprimant $\mathrm{Cre}$, ce qui permet la transcription du transgène.
}

reproductibilité, et une possibilité de suivre la progression de la tumeur et l'effet du traitement [9]. Ces modèles se raffinent progressivement pour en repousser les limitations [10]. Ils se sont cependant déjà révélés fructueux dans l'étude des cancers du côlon, du sein, de la peau, des cancers oculaires, etc. Ainsi, les traitements par le taxol ont été mis au point, entre autres, grâce à des modèles chimères souris-homme. Actuellement, l'efficacité de certains traitements anti-cancéreux est en premier lieu testée sur des modèles souris, ce qui a conduit l'université de Harvard (Beth Israel Deaconess Medical Center et Harvard Medical School) à développer un centre dédié, le «Mouse hospital - preclinical murine pharmacogenetics core $»^{3}$.

Mais, comme pour tout organisme-modèle, il existe des domaines pour lesquels la souris n'est pas un modèle valide: par exemple, les mécanismes de réponse à l'ischémie et à l'hypoxie sont différents de ceux de l'homme [11] ou encore ceux impliqués dans la réponse à un choc septique [12], ce qui nécessite de développer ces champs d'investigation à l'aide d'autres modèles qu'ils soient in vivo ou in vitro.

\section{Le modèle rat}

Le rat (Rattus norvegicus) est l'autre espèce de rongeur utilisée en recherche biomédicale, même si son importance numérique est moindre que celle de la souris. C'est aussi une espèce de petite taille qui présente l'avantage, par rapport à la souris, d'être plus proche physiologiquement de l'homme, conférant ainsi aux résultats des recherches une meilleure performance translationnelle [13]. Ainsi, le rat représente le modèle de choix pour tester l'efficacité ou la toxicité de molécules avant de passer aux essais pré-cliniques chez l'homme.

Le rat est plus grand que la souris (environ 10 fois plus). Il est ainsi plus facile à manipuler, par exemple pour des gestes chirurgicaux. Sa taille autorise aussi des volumes de prélèvement plus importants et une imagerie plus facile qu'avec la souris. II a une durée de vie plus longue que la souris (de l'ordre de deux ans) permettant des études sur les mécanismes du vieillissement ou les phénomènes neurodégénératifs [14]. Sa neuroanatomie est proche de celle de l'homme en termes de fonction des territoires cérébraux et de connectivité. Sa plus grande taille facilite la possibilité de mettre en œuvre des techniques d'optogénétique permettant de stimuler très précisément certains réseaux neuronaux. Cette espèce présente également une très grande variété de profils

${ }^{3}$ II y a deux grands centres en France : à l'IGBMC et au CIML : I'Institut Clinique de
la Souris : http://www.ics-mci.fr/en/ et le Centre d'immunophénomique : https:// ciphe.marseille.inserm.fr/ 
de comportements, ce qui la rend particulièrement intéressante pour les études sur les mécanismes de l'apprentissage et de la mémorisation ainsi que pour les mécanismes de la récompense et de l'addiction $[15,16]$. On peut prendre pour exemple le Prix Nobel de Physiologie ou Médecine 2014, attribué à 0'Keefe, Moser et Moser, mettant en évidence les cellules de lieu dans l'hippocampe ${ }^{4}$, ou encore ce modèle de binge drinking (consommation d'alcool excessive en un temps court), le premier modèle pré-clinique réaliste, élaboré par Jeanblanc [17]. Le modèle rat a également permis des avancées dans l'étude des cancers. Ainsi, pour les cancers de la prostate qui sont classés au $2^{\mathrm{e}}$ rang dans les cancers de l'homme avec plus de 300000 décès en 2012, le rat s'avère avoir une sensibilité aux hormones proche de la sensibilité humaine et il présente un développement histologique des tumeurs similaire. Sa taille permet en outre de suivre par imagerie la carcinogenèse [18]. Dans le cas des études portant sur l'endométriose, le premier modèle qui a été développé l'a été chez le rat [19]. Il a permis de nombreuses avancées dans la compréhension de la physiopathologie, notamment en démontrant le rôle des métalloprotéases matricielles (MMP) dans l'établissement des lésions ectopiques [20]. Ce modèle est actuellement utilisé pour explorer les effets d'une exposition à des perturbateurs endocriniens comme facteurs étiologiques de l'endométriose [21].

Plusieurs modèles de rats mutants spontanés sont utilisés, notamment dans le domaine de la recherche cardio-vasculaire avec la souche SHR (spontaneously hypertensive rat) atteinte d'hypertension artérielle, dans celui de la physiologie rénale avec la souche Brattleboro atteinte de diabète insipide d'origine centrale, ou enfin dans le domaine de l'obésité avec la souche Zucker.

Les modèles rats ont souffert pendant longtemps des difficultés à mettre en œuvre les outils de l'édition du génome dans cette espèce. Les premiers rats déficients en un gène (knock-out) ont été générés en 2010 par recombinaison de CSE [22]. Cependant, les cellules ES de rat sont plus fragiles que celles de la souris, ce qui limite leur usage. Les techniques d'édition (ou modification) du génome sont maintenant disponibles chez le rat (zinc finger nucleases [ZFN], transcription activator-like effector nucleases [TALEN], CRISPR/Cas9) [23], ce qui suggère que la transgénèse devrait se développer maintenant très rapidement.

\section{Conclusion}

Comme nous venons de le voir dans ces différents exemples, le recours aux modèles animaux reste indispensable à la production de connaissances dans différents champs disciplinaires de la biologie. Les rongeurs constituent des organismes-modèles présentant de multiples avantages, notamment liés à leur physiologie proche de celle de l'homme. Cependant, ils présentent aussi leurs limites, en termes de zootechnie, de diversité génétique ou d'infectiosité. Ces limites peuvent être contournées par l'usage d'un éventail plus large de modèles expérimentaux, autres organismes-modèles, méthodes alternatives, ou méthodes d'investigation non invasives chez l'homme lui-même, la complémentarité entre

${ }^{4}$ Les cellules de lieu sont des neurones de l'hippocampe dont le champ récepteur (dit champ de lieu) est défini par une zone spatiale donnée sur une carte cognitive, qui est une carte mentale de l'environnement. différentes approches constituant en outre une source de robustesse des résultats expérimentaux. $\diamond$

Mice, rats and men: how rodent models are still required to produce knowledge

\section{LIENS D’INTÉRÊT}

Les auteurs déclarent n'avoir aucun lien d'intérêt concernant les données publiées dans cet article.

\section{RéFÉRENCES}

1. Académie Vétérinaire de France. Rapport de la commission relation hommeanimaux sur la recherche scientifique et l'expérimentation animale : état de la question. Approuvé en mai 2012.

2. Russell WMS, Burch RL. The principles of humane experimental technique. Londres: Methuen, $1959: 238 \mathrm{p}$.

3. Ministère de L'enseignement supérieur, de la recherche et de l'innovation. Utilisation des animaux à des fins scientifiques dans les établissements utilisateurs français. Enquête statistique, 2016.

4. Church RJ, Gatti DM, Urban TJ, et al. Sensitivity to hepatotoxicity due to epigallocatechin gallate is affected by genetic background in diversity outbred mice. Food Chem Toxicol $2015 ; 76$ : 19-26.

5. Malissen M, Vallet-Erdtmann V, Guillou F, et al. Les modèles animaux en recherche biomédicale. Biofutur $2010 ; 29: 34-8$.

6. Ogunniyi AD, Kopecki Z, Hickey $\varepsilon \varepsilon$, et al. Bioluminescent murine models of bacterial sepsis and scald wound infections for antimicrobial efficacy testing. PLoS One 2018 ; 13 : e0200195.

7. Moldovan $M$, Alvarez $S$, Rothe $C$, et al. An in vivo mouse model to investigate the effect of local anesthetic nanomedicines on axonal conduction and excitability. Front Neurosci $2018 ; 12: 494$

8. Gillis CM, Reber LL. Un nouveau modèle de souris pour comprendre le rôle des neutrophiles. Med Sci (Paris) 2018 ; $34: 339-43$.

9. Kohnken R, Porcu P, Mishra A. Overview of the use of murine models in leukemia and lymphoma research. Front Oncol $2017 ; 7: 22$.

10. Talmadge JE, Singh RK, Fidler IJ, et al. Murine models to evaluate novel and conventional therapeutic strategies for cancer. Am J Pathol 2007 ; 170 : 793-804.

11. Haouzi P. Murine models in critical care research. Crit Care Med 2011 ; 39 : 2290-3.

12. Radermacher P, Haouzi P. A mouse is not a rat is not a man: species-specific metabolic responses to sepsis - a nail in the coffin of murine models for critical care research? Intensive Care Med Exp 2013; $1: 26$.

13. Lee JG, Sung $\mathrm{YH}$, Baek IJ. Generation of genetically-engineered animals using engineered endonucleases. Arch Pharm Res 2018; 41 : 885-97.

14. Creed RB, Goldberg MS. New developments in genetic rat models of Parkinson's disease. Mov Disord 2018 ; 33 : 717-29.

15. Ahmed SH. Trying to make sense of rodents' drug choice behavior. Prog Neuropsychopharmacol Biol Psychiatry 2018 ; 87 : 3-10.

16. Slaney CL, Hales CA, Robinson ESJ. Rat models of reward deficits in psychiatric disorders. Curr Opin Behav Sci 2018 ; 22 : 136-42.

17. Jeanblanc J, Rolland B, Gierski F, et al. Animal models of binge drinking, current challenges to improve face validity. Neurosci Biobehav Rev 2018 ; May 5. pii: S0149-7634(18)30123-4.

18. Nascimento-Gonçalves $\varepsilon$, Faustino-Rocha Al, Seixas F, et al. Modelling human prostate cancer: Rat models. Life Sci 2018 ; 203 : 210-24.

19. Vernon MW, Wilson $\varepsilon A$. Studies on the surgical induction of endometriosis in the rat. Fertil Steril $1985 ; 44: 684-94$.

20. Sharpe-Timms KL. Using rats as a research model for the study of endometriosis. Ann Ny Acad Sci 2002 ; 955 : 318-27.

21. Bruner-Tran KL, Mokshagundam S, Herington JL, et al. Rodent models of experimental endometriosis: identifying mechanisms of disease and therapeutic targets. Curr Womens Health Rev 2018 ; 14 : 173-88.

22. Tong $C$, Li P, Wu NL, et al. Production of $p 53$ gene knockout rats by homologous recombination in embryonic stem cells. Nature $2010 ; 467: 211-3$.

23. Li D, Qiu Z, Shao Y, et al. Heritable gene targeting in the mouse and rat using a CRISPR-Cas system. Nat Biotechnol 2013 ; $31: 681-3$.

24. Gilgenkrantz H. La révolution des CRISPR est en marche. Med Sci (Paris) $2014 ; 30: 1066-9$

\section{TIRÉS À PART}

H. Hardin-Pouzet 